a. Arctic

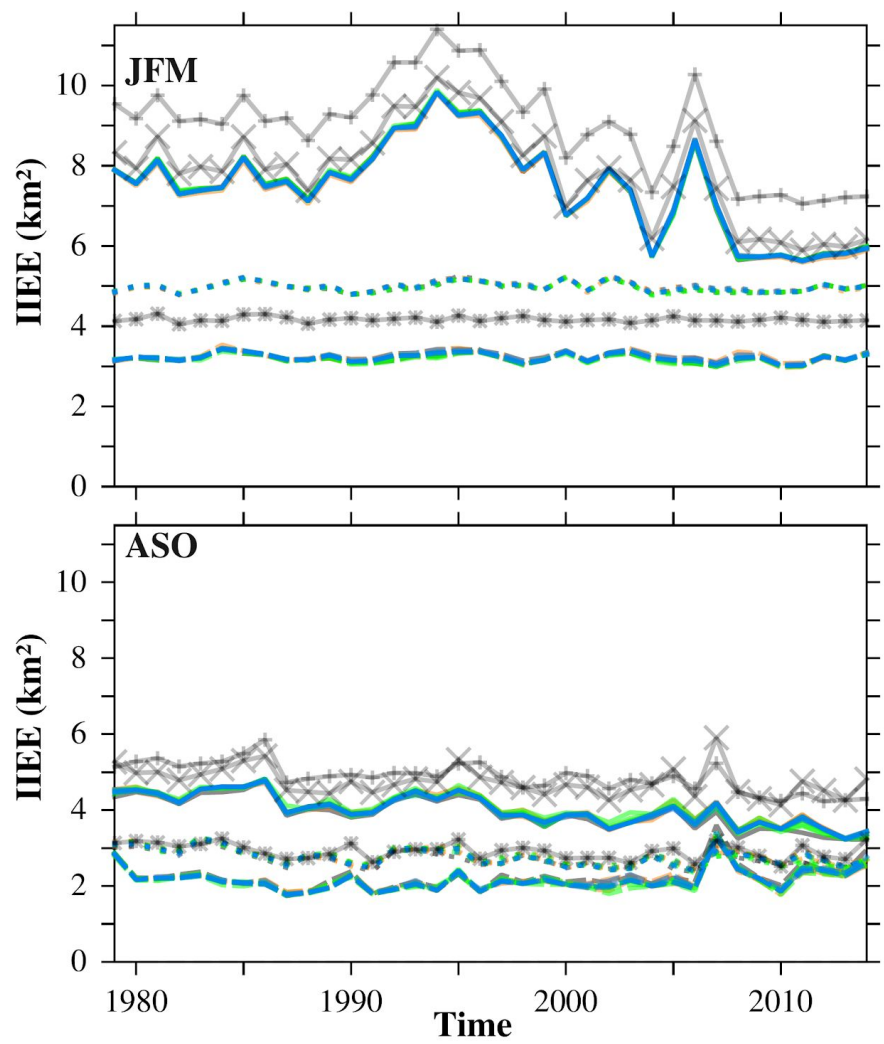

b. Antarctica

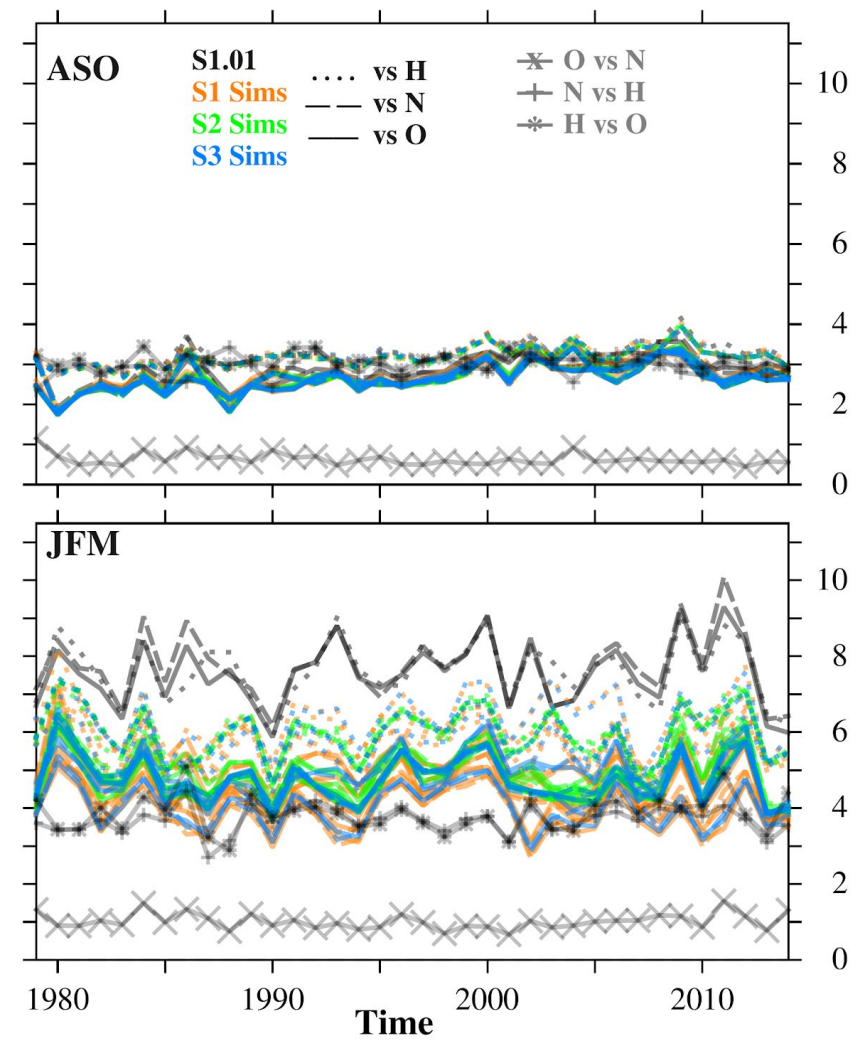

Fig. 1: (a) Arctic and (b) Antarctic integrated ice edge error (IIEE; in $\mathrm{km}^{2}$ ) between the simulated SIC in the S1 (red lines), S2 (green lines), and S3 (blue lines) ITD configurations and the observed SIC in HadISST (dotted lines), NSIDC (dashed lines), and OSISAF (solid lines), in JFM (top) and ASO (bottom). Also, IIEE is shown between OSI SAF and NSDIC SIC (gray crosses), NSIDC and HadISST SIC (gray pluses), and HadISST and OSI SAF SIC (gray asterisks). The IIEE is calculated as the integrated area where simulations and observations disagree on ice concentration above $15 \%$ [Goessling et al., 2016]. 

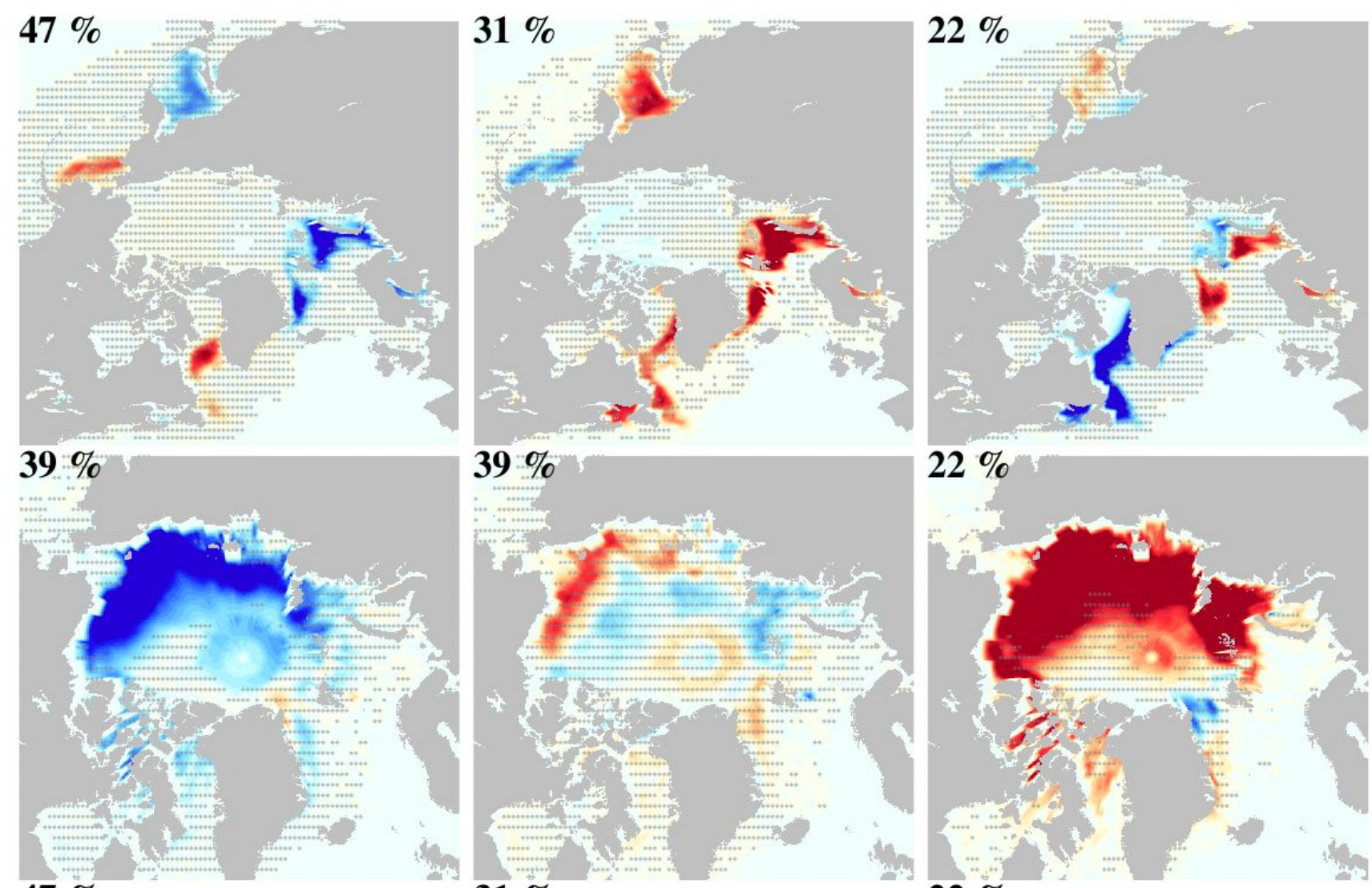

$47 \%$

$39 \%$

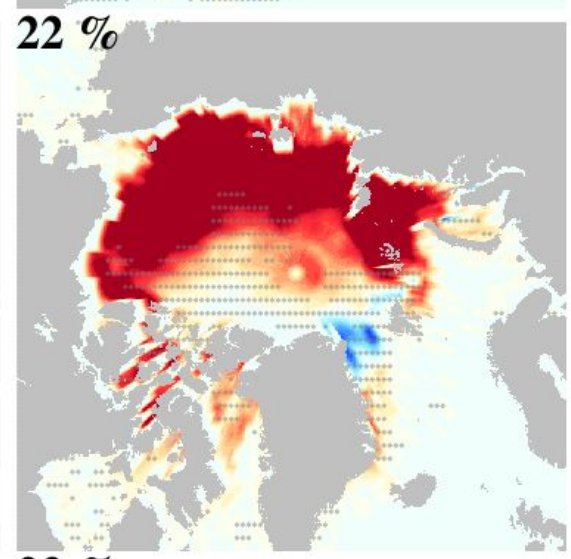

$31 \%$

$22 \%$
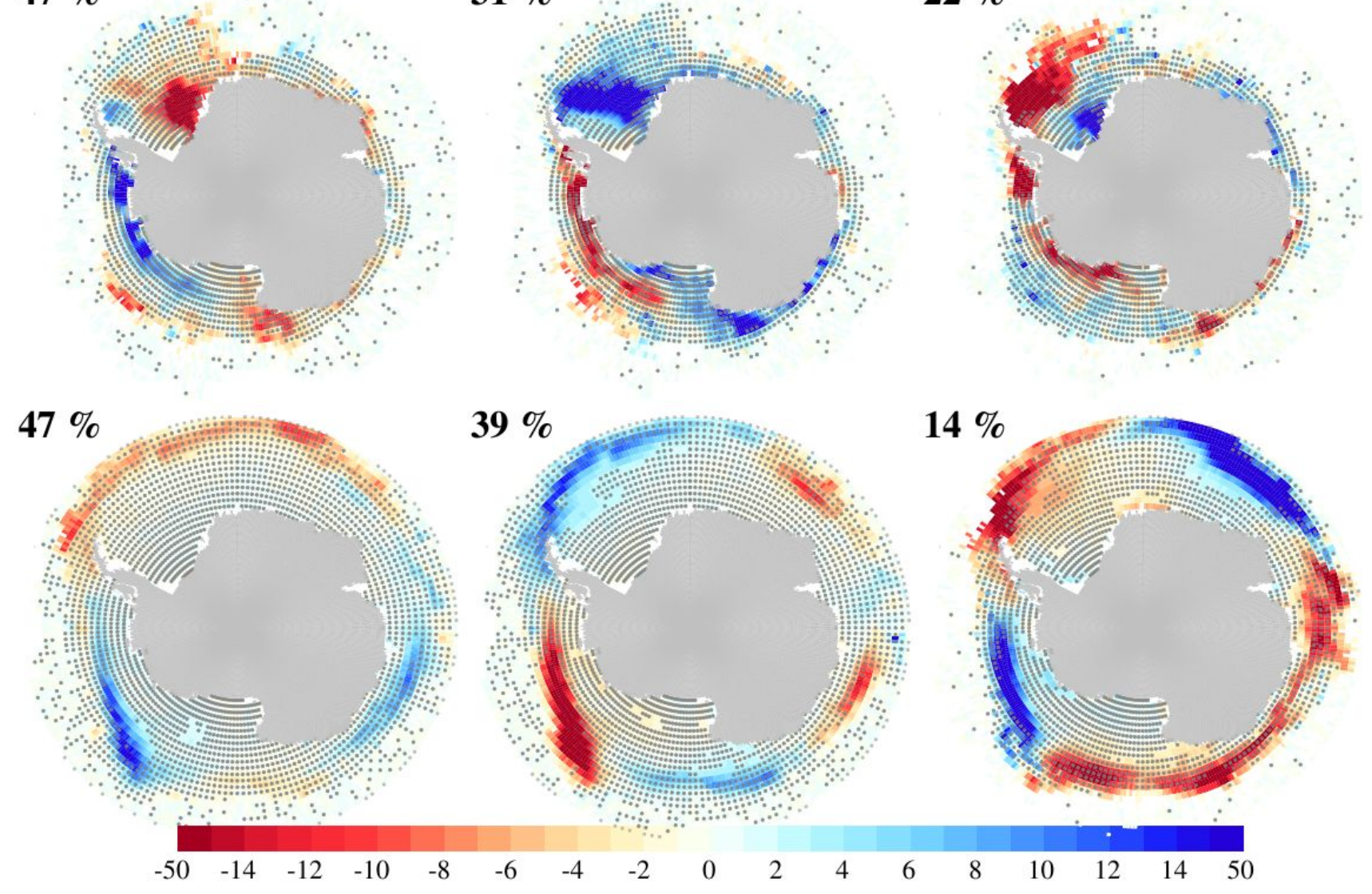

Supp. Fig. 2: Cluster patterns of Arctic (top two rows) and Antarctic (bottom two rows) SIC anomalies (shading; in \% of area) in NSDIC in JFM (first and third rows) and ASO (second and fourth rows). The area is zoomed in over the Arctic in ASO (second row) for a better view of the central Arctic. Clusters are calculated from the full SIC field without detrending. Stippling masks statistically non-significant anomalies at the $5 \%$ level. The shading color scale of the SIC anomalies is adapted for a better view of values in the range $\pm 15 \%$. 

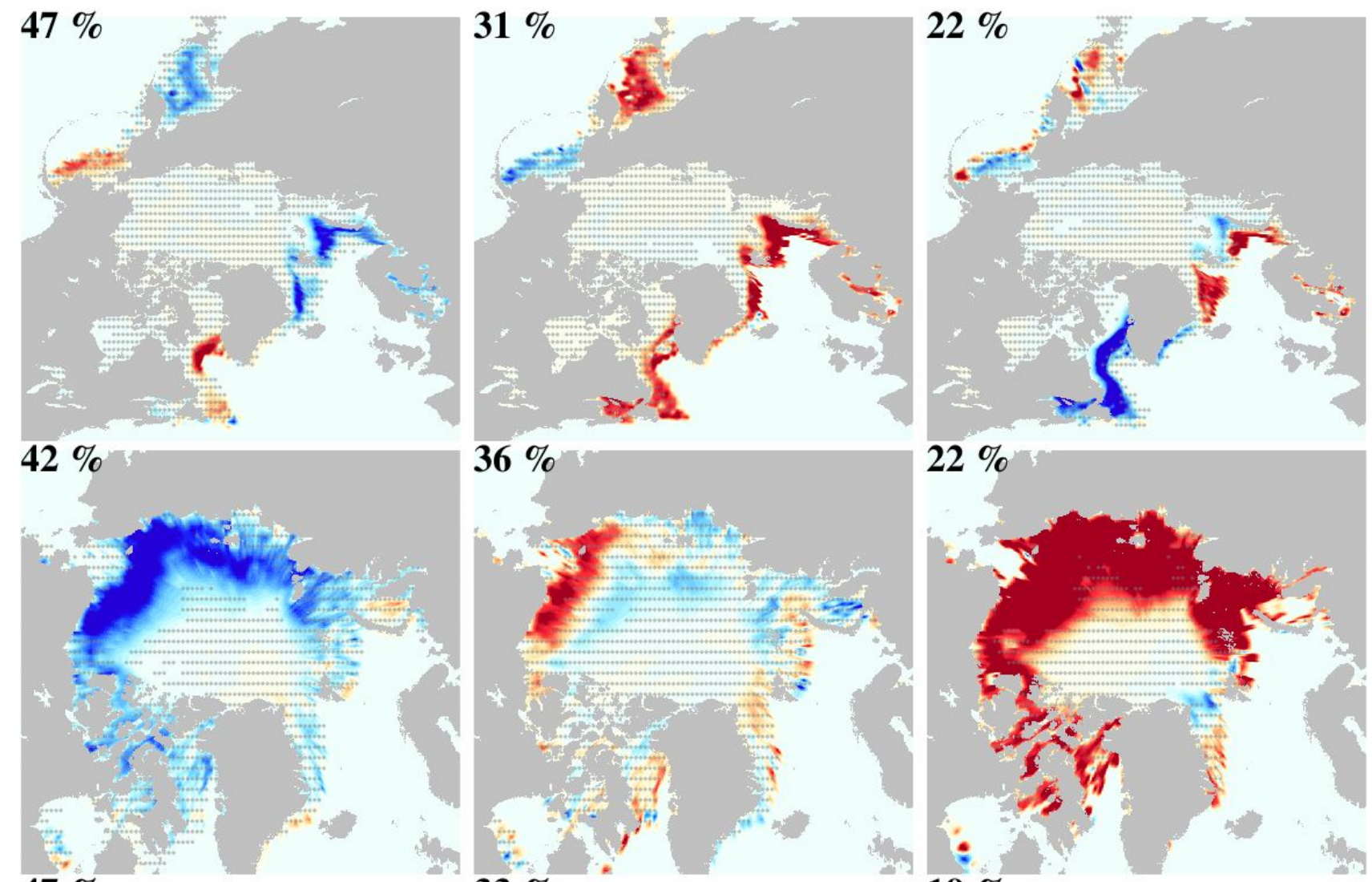

$36 \%$

$22 \%$

$47 \%$

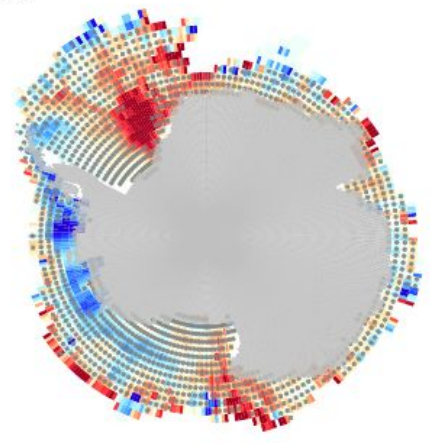

$47 \%$

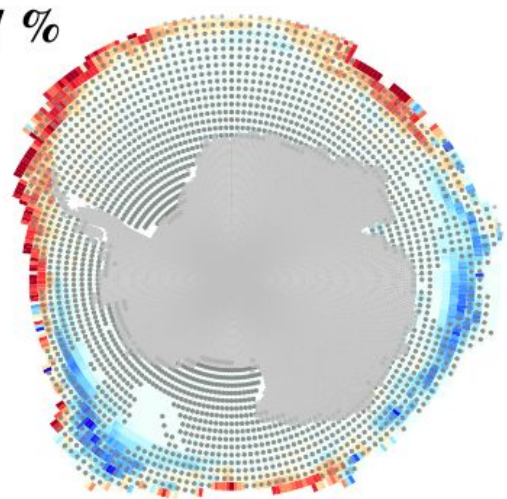

$33 \%$

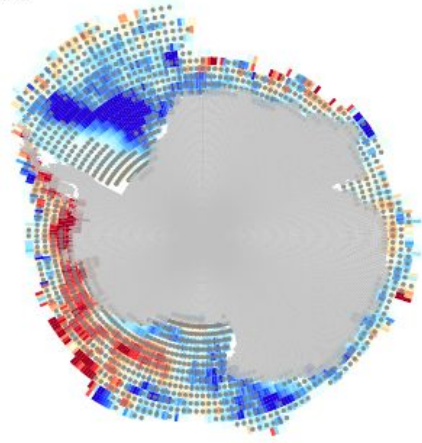

$33 \%$

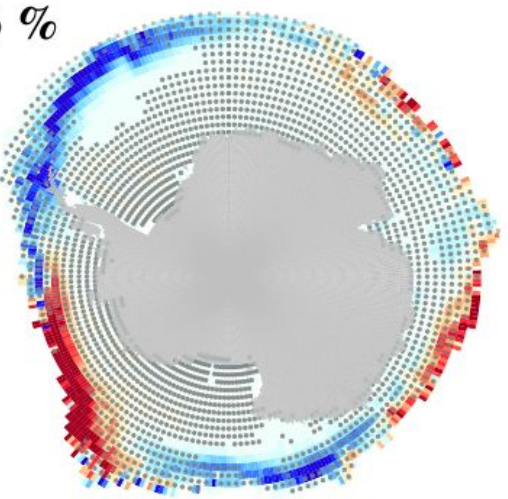

$19 \%$

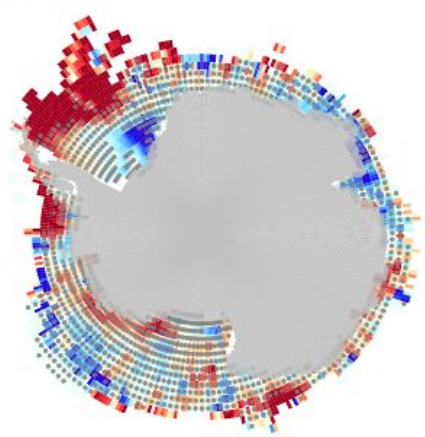

$19 \%$

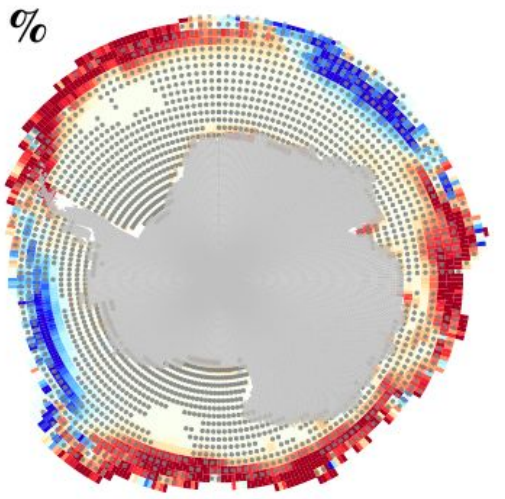

$\begin{array}{lllllllllllllllll}-50 & -14 & -12 & -10 & -8 & -6 & -4 & -2 & 0 & 2 & 4 & 6 & 8 & 10 & 12 & 14 & 50\end{array}$

Supp. Fig. 3: As in Supp. Fig. 2 but for HadISST 


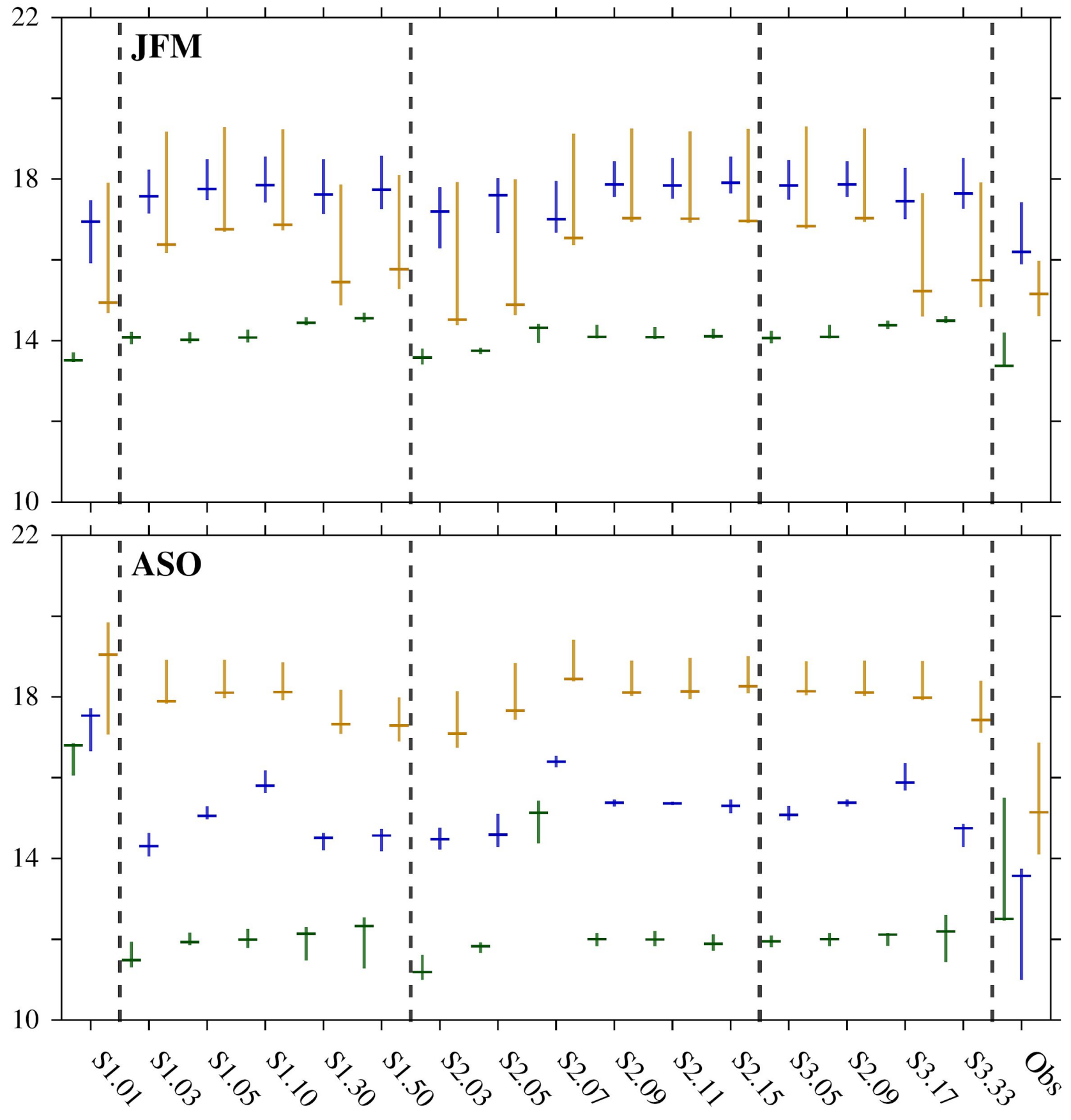

Supp. Fig. 4: Boxplot of the root mean square error (in $\%$ of area) between the simulated and observed clusters and across the three satellite observational products of Arctic SIC in JFM (top) and ASO (bottom). Results for the first, second, and third clusters are in green, blue, and orange respectively. For each ITD configuration, three values are calculated with OSI SAF, NSIDC, and HadISST. 

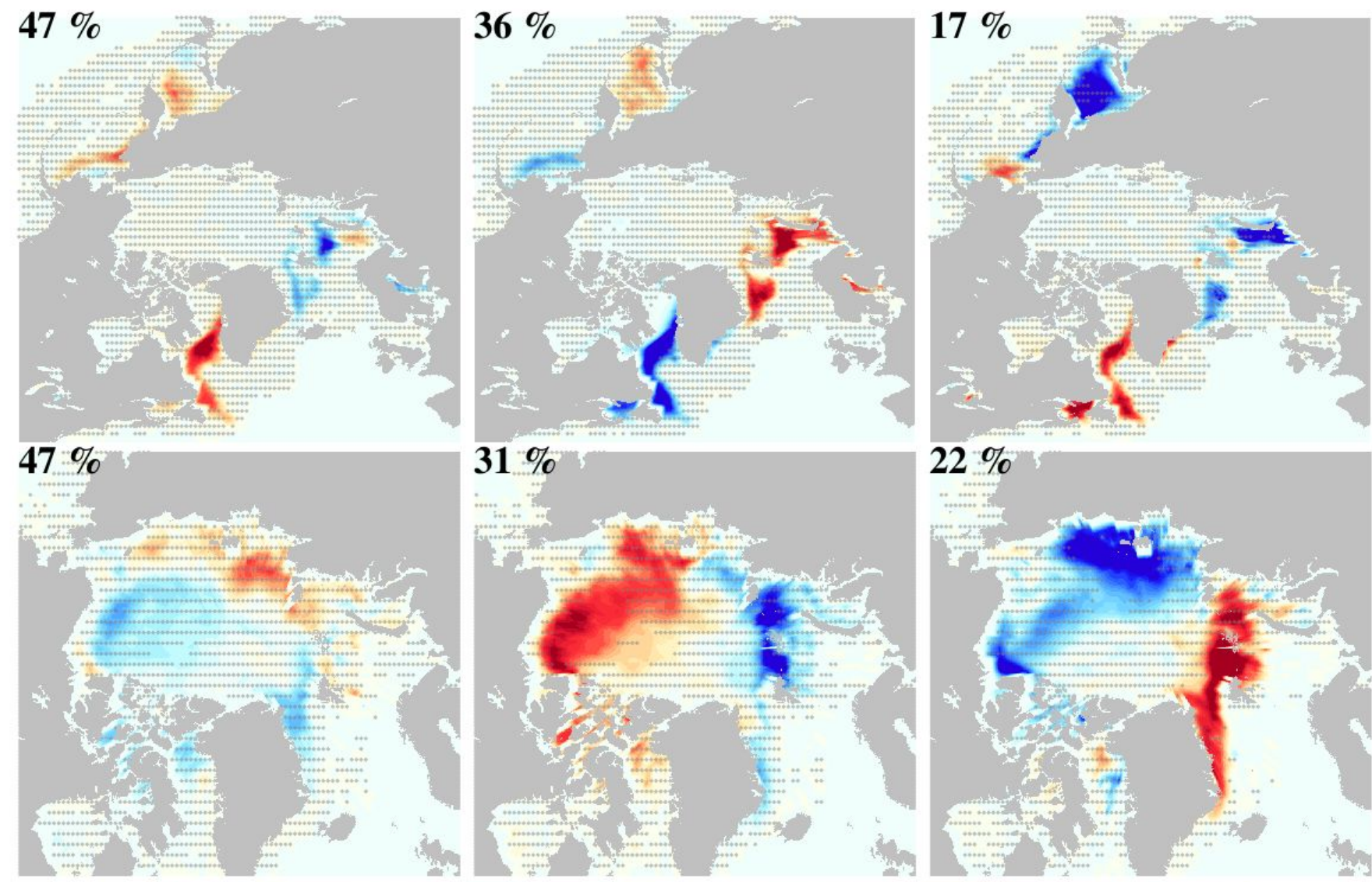

$22 \%$
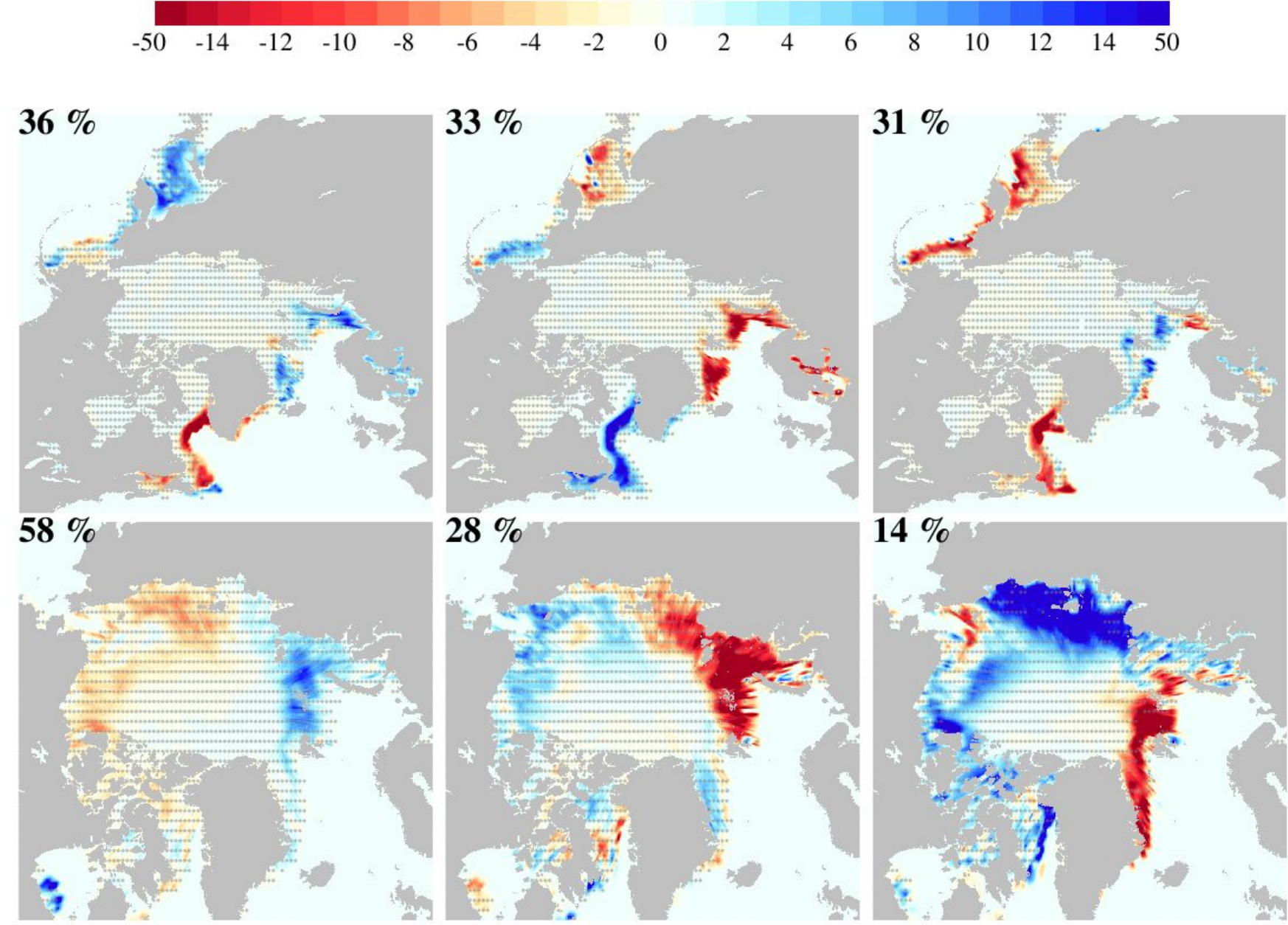

\section{$28 \%$}

\section{$14 \%$}
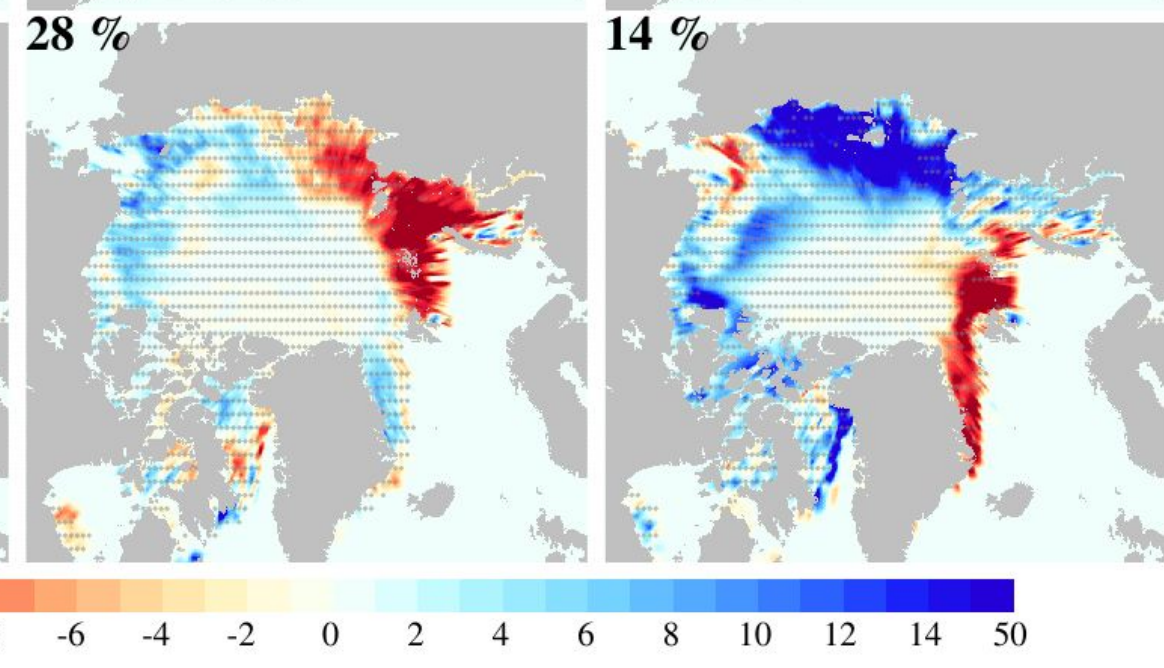

Supp. Fig. 5: As in Supp. Fig. 2 but for NSIDC and HadISST data (top and bottom two rows respectively) after detrending with a 2nd degree polynomial and in the Arctic only. 

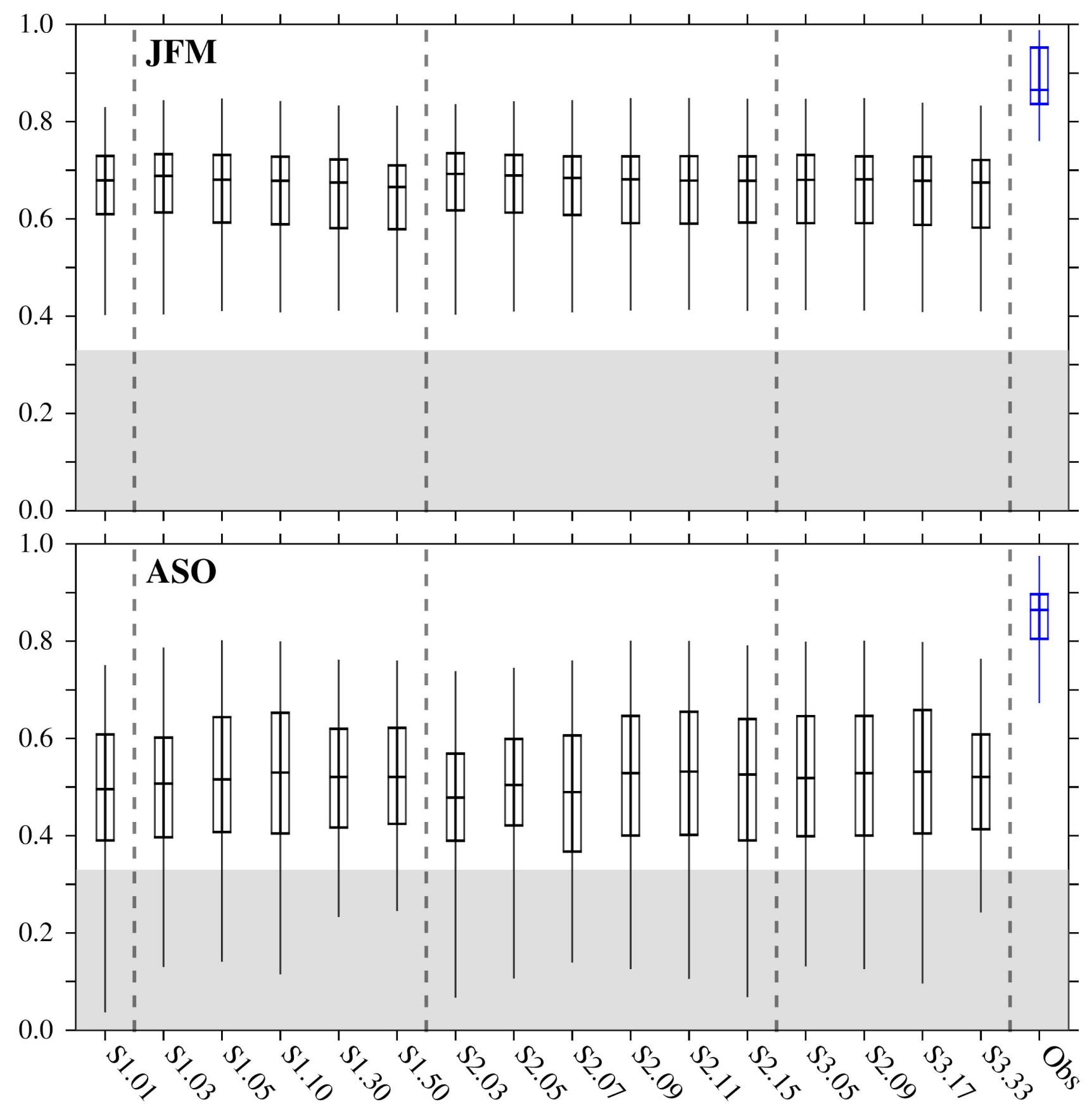

Supp. Fig. 6: Boxplot of spatial correlation coefficients between the simulated and observed anomaly fields (gray) and across the three satellite observational products (blue) of Arctic SIC in JFM (top) and ASO (bottom) between 1979 and 2014. For each ITD configuration, a distribution of 36 spatial correlation coefficients, corresponding to 36 years, is computed from the spatial correlation coefficients between its anomalies and those in OSISAF, NSIDC, and HadISST. Similarly, three 36-coefficient distributions are obtained between the three observational products. From these distributions, the maximum and minimum values, the 25th, 50th (media), and 75th percentiles are calculated and plotted. Gray shading masks statistically non-significant coefficients below 0.39 value, which corresponds with the minimum value across all the computations that is statistically significant at the 5\% level, accounting for effective degrees of freedom and spatial autocorrelation. Dashed vertical lines separate between results in the simulation with one category (S1.01), in the different STD configurations (S1, S2, and S3), and in the observations. 

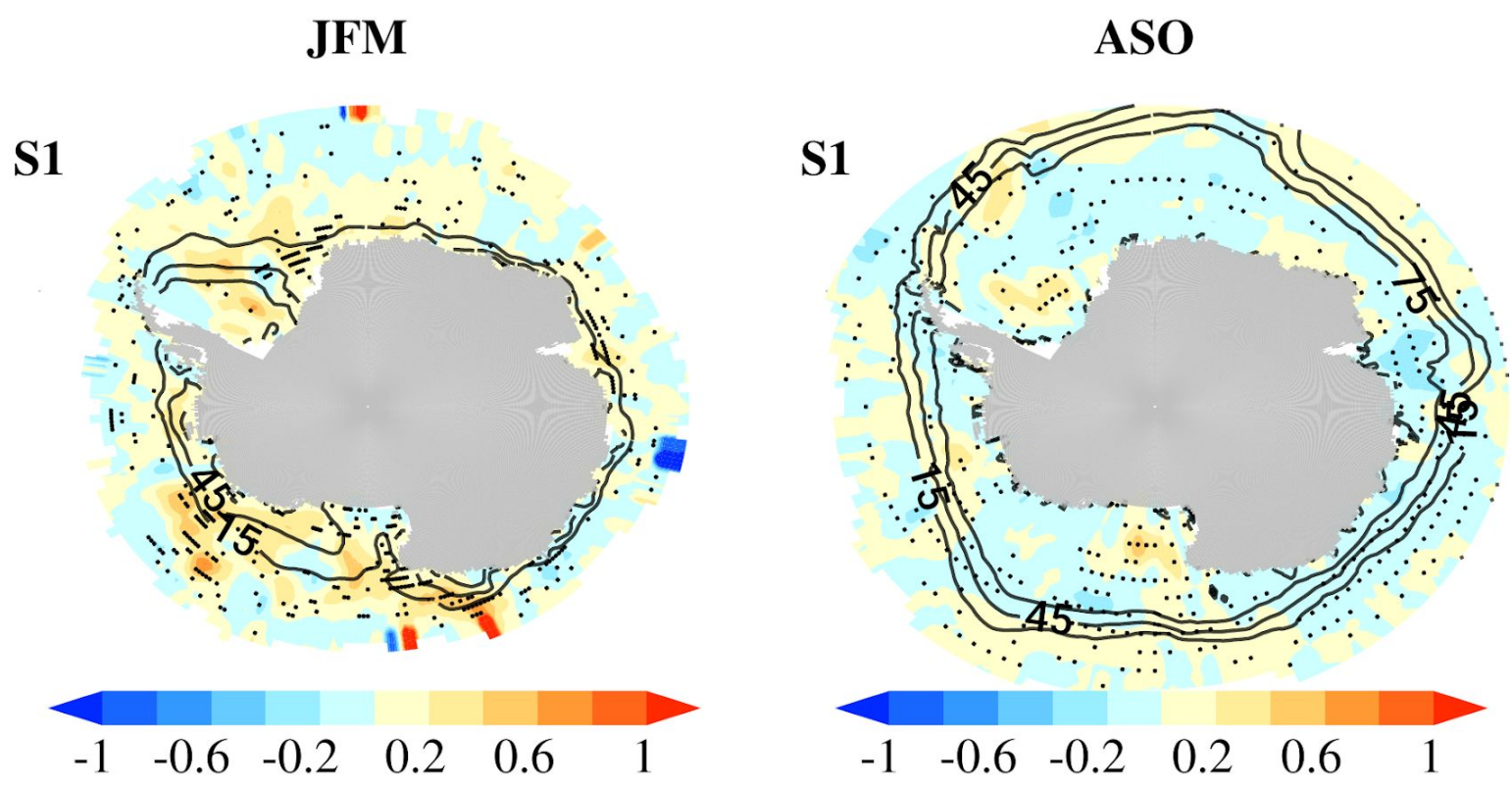

S2

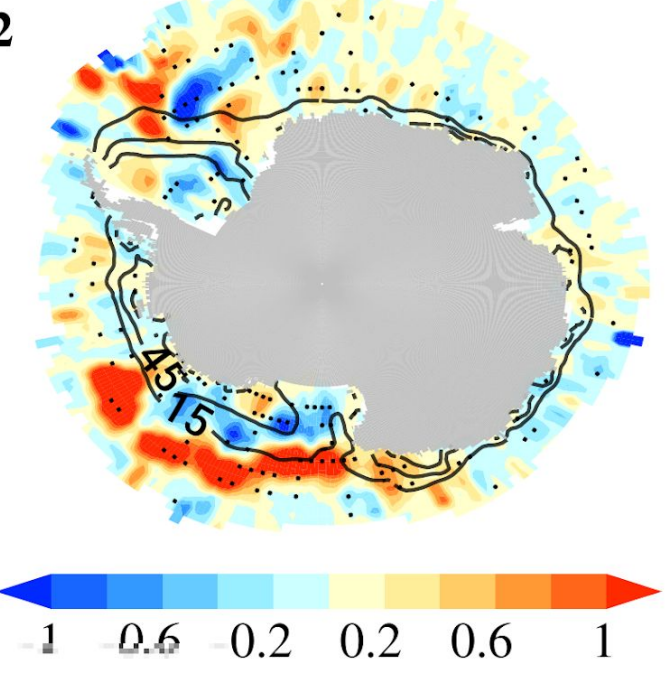

\section{S2}

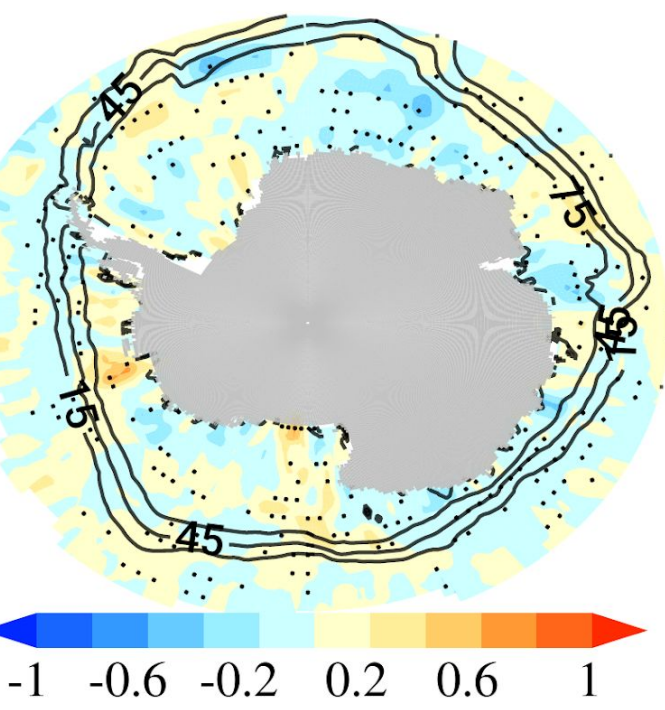

$\$ 3$
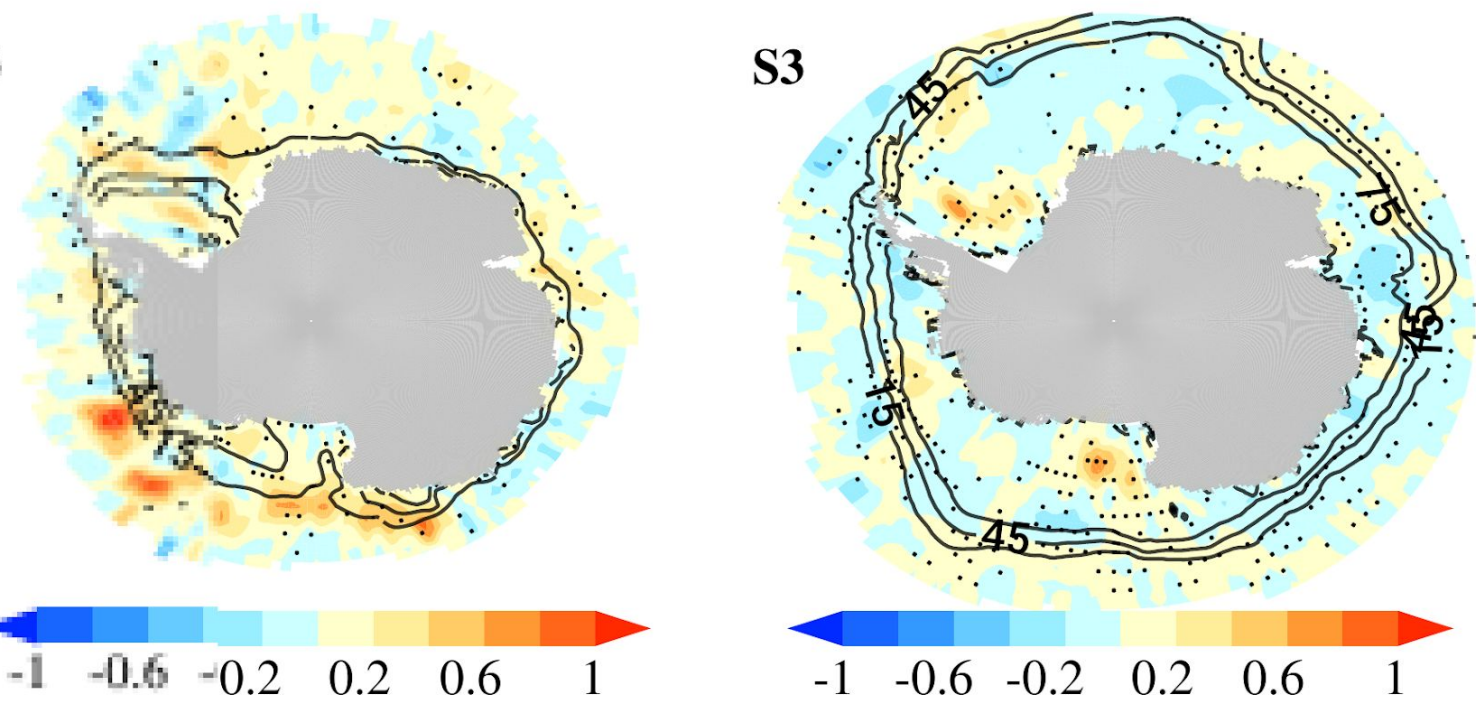

Supp. Fig. 7: As in Fig. 11, but for in Antarctica. Contours are climatological SIC for the period 1979-2014 in OSI SAF. 